\title{
Performance Comparison of various levels of Fusion of Multi-focused Images using Wavelet Transform
}

\author{
K. Kannan \\ Professor \& Head/ECE \\ Kamaraj College of Engineering \& \\ Technology, Virudhunagar, India
}

\author{
S. Arumuga Perumal \\ Professor \& Head \\ Department of Computer Science \\ S. T. Hindu College, Nagarcoil, India
}

\author{
K. Arulmozhi \\ Principal, Kamaraj College of \\ Engineering \& Technology, SPGC \\ Nagar, Virudhunagar, India
}

\begin{abstract}
The fast development of digital image processing leads to the growth of feature extraction of images which leads to the development of Image fusion. Image fusion is defined as the process of combining two or more different images into a new single image retaining important features from each image with extended information content. There are two approaches to image fusion, namely Spatial Fusion and Transform fusion. In Spatial fusion, the pixel values from the source images are directly summed up and taken average to form the pixel of the composite image at that location. Transform fusion uses transform for representing the source images at multi scale. The most common widely used transform for image fusion at multi scale is Wavelet Transform since it minimizes structural distortions. But, wavelet transform suffers from lack of shift invariance \& poor directionality and these disadvantages are overcome by Stationary Wavelet Transform and Dual Tree Wavelet Transform. The conventional convolution-based implementation of the discrete wavelet transform has high computational and memory requirements. Lifting Wavelets has been developed to overcome these drawbacks. The Multi-Wavelet Transform of image signals produces a non-redundant image representation, which provides better spatial and spectral localization of image formation than discrete wavelet transform. And there are three levels of image fusion namely Pixel level, Area level and region level. This paper evaluates the performance of all levels of multi focused image fusion of using Discrete Wavelet Transform, Stationary Wavelet Transform, Lifting Wavelet Transform, Multi Wavelet Transform, Dual Tree Discrete Wavelet Transform and Dual Tree Complex Wavelet transform in terms of various performance measures.
\end{abstract}

\section{Keywords}

Image Fusion, Discrete Wavelet Transform, Stationary Wavelet Transform, Lifting Wavelet Transform, Multi Wavelet Transform, Dual Tree Discrete Wavelet Transform and Dual Tree Complex Wavelet transform.

\section{INTRODUCTION}

Image fusion is defined as the process of combining two or more different images into a new single image retaining important features from each image with extended information content. For example, IR and visible images may be fused as an aid to pilots landing in poor weather or microwave and visible images may be fused to detect weapons or CT and MRI images may be fused as an aid to medical diagnosis. The fusion process must satisfy the following requirements such as it should preserve all relevant information in the fused image, should suppress noise and should minimize any artifacts in the fused image. There are two approaches to image fusion, namely Spatial Fusion and Transform fusion. In Spatial fusion, the pixel values from the source images are summed up and taken average to form the pixel of the composite image at that location [1]. Transform fusion use pyramid or Wavelet Transform (WT) for representing the source image at multi scale. The most widely used transform for image fusion at multi scale is Discrete Wavelet Transform (DWT) since it minimizes structural distortions. But, DWT suffers from lack of shift invariance \& poor directionality and these disadvantages are overcome by Stationary Wavelet Transform (SWT), Dual Tree Discrete Wavelet Transform (DTDWT) and Dual Tree Complex Wavelet Transform (DTCWT). The conventional convolutionbased implementation of the discrete wavelet transform has high computational and memory requirements. Lifting Wavelet Transform (LWT) has been developed to overcome these drawbacks. The Multi-Wavelet Transform (MWT) of image signals produces a non-redundant image representation, which provides better spatial and spectral localization of image formation than discrete wavelet transform There are three levels in multi resolution fusion scheme namely Pixel level fusion, area level fusion and region level fusion. The performance measures which can be computed independently of the subsequent tasks express the successfulness of an image fusion technique by the extent that it creates a composite image that retains salient information from the source images while minimizing the number of artifacts or the amount of distortion that could interfere with interpretation. In this paper, it is proposed to evaluate the performance of all levels of multi focused image fusion using DWT, SWT, LWT, MWT, DTDWT and DTCWT in terms of various performance measures like Root Mean Square Error (RMSE), Peak to Signal Noise Ratio (PSNR), Quality Index (QI) and Normalized Weighted Performance Metric (NWPM).

\section{WAVELET TRANSFORM THEORY}

Wavelet theory and wavelet analysis is a relatively recent branch of mathematics. The first wavelet was developed by Alfred Haar in 1909. The Haar wavelet belongs to the group of wavelets known as Daubechies wavelets, which are named after Ingrid Daubechies, who proved the existence of wavelet families whose 
scaling functions have certain useful properties, namely compact support over an interval, at least one nonvanishing moment, and orthogonal translates. Because of its simplicity, the Haar wavelet is useful for illustrating the basic concepts of wavelet theory but has limited utility in applications. The wavelet function $\psi(\mathrm{x})$ and scaling function $\phi(\mathrm{x})$ of Haar wavelet is presented in figure1.

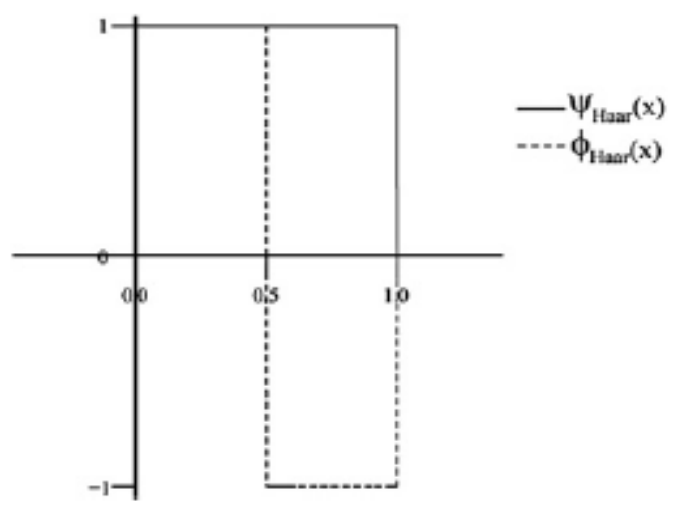

Figure 1. The wavelet and scaling function of Haar wavelet

Various researchers further developed the concept of wavelets over the next half century but it was not until the 1980's that the relationships between quadrature mirror filters, pyramid algorithms, and orthonormal wavelet bases were discovered, allowing wavelets to be applied in signal processing. Over the past decade, there has been an increasing amount of research into the applications of wavelet transforms to remote sensing, particularly in image fusion. It has been found that wavelets can be used to extract detail information from one image and inject it into another, since this information is contained in high frequencies and wavelets can be used to select a set of frequencies in both time and space. The resulting merged image, which can in fact be a combination of any number of images, contains the best characteristics of all the original images.

Wavelets can be described in terms of two groups of functions: wavelet functions and scaling functions. It is also common to refer to them as families: the wavelet function is the "mother" wavelet, the scaling function is the "father" wavelet, and transformations of the parent wavelets are "daughter" and "son" wavelets. Generally, a wavelet family is described in terms of its mother wavelet, denoted as $\psi(\mathrm{x})$. The mother wavelet must satisfy certain conditions to ensure that its wavelet transform is stably invertible. These conditions are:

$$
\begin{aligned}
& \int|\Psi(x)|^{2} d x=1 \\
& \int|\Psi(x)| d x<\infty \\
& \int \Psi(x) d x=0
\end{aligned}
$$

The conditions specify that the function must be an element of $\mathrm{L}^{2}(\mathrm{R})$, and in fact must have normalized energy, that it must be an element of $L^{1}(R)$, and that it have zero mean. The third condition allows the addition of wavelet coefficients without changing the total flux of the signal. Other conditions might be specified according to the application. For example, the wavelet function might need to be continuous, or continuously differentiable, or it might need to have compact support over a specific interval, or a certain number of vanishing moments. Each of these conditions affects the results of the wavelet transform. To apply a wavelet function, it must be scaled and translated. Generally, a normalization factor is also applied so that the daughter wavelet inherits all of the properties of the mother wavelet. A daughter wavelet $\psi_{\mathrm{a}, \mathrm{b}}(\mathrm{x})$ is defined by the equation,

$$
\Psi_{a, b}(x)=a^{-1 / 2} \Psi((x-b) / a)
$$

where $a, b \in R$ and $a \neq 0$; $a$ is called the scaling or dilation factor and $b$ is called the translation factor. In most practical applications it is necessary to place limits on the values of $a$ and $b$. A common choice is $a=2^{-j}$ and $b=2^{-j} k$, where $j$ and $k$ are integers. The resulting equation is

$$
\Psi_{j, k}(x)=2^{1 / 2} \Psi\left(2^{j} x-k\right)
$$

This choice for dilation and translation factors is called a dyadic sampling. Changing $\mathrm{j}$ by one corresponds to changing the dilation by a factor of two, and changing $\mathrm{k}$ by one corresponds to a shift of $2^{-j}$. Figure 3 uses the Haar wavelet to illustrate the relationship of daughter wavelets to the mother wavelet and the effect of varying dilation and translation for both the general equation and the dyadic equation. The mother wavelet is $\psi_{1,0}(\mathrm{x})$ in Fig. 3a and $\psi^{0,0}(\mathrm{x})$ in Fig. 3b. Non-integer values are used for $\mathrm{j}$ and $\mathrm{k}$ in one example in Fig. $3 \mathrm{~b}$ to allow direct comparison with $\psi_{0.5},{ }_{1.5}(\mathrm{x})$ in Fig. 3a. In discrete wavelet transforms, a scaling function, or father wavelet, is needed to cover the low frequencies. If the mother wavelet is regarded as a high pass filter then the father wavelet, denoted as $\varphi(\mathrm{x})$, should be a low pass filter. To ensure that this is the case, it cannot have any vanishing moments. It is useful to specify that, in fact, the father wavelet have a zeroth moment, or mean, equal to one:

$$
\int \phi(x) d x=1
$$

In mathematical terms, $\phi(x)$ is chosen so that the set $\{\phi(x-k)$, $\mathrm{k} \in \mathrm{Z}\}$ forms an orthonormal basis for the reference space V0. A subspace $\mathrm{Vj}$ is spanned by $\left\{\phi_{j, k}(x)=2^{1 / 2} \phi\left(2^{j} x-k\right), k \in Z\right\}$. Multiresolution analysis makes use of a closed and nested sequence of subspaces $\{\mathrm{Vj}\} \mathrm{j} \in \mathrm{Z}$, which is dense in $\mathrm{L}^{2}(\mathrm{R})$ : each subsequent subspace is at a higher resolution and contains all the subspaces at lower resolutions. Since the father wavelet is in V0, it, as well as the mother wavelet, can be expressed as linear combinations of the basis functions for $\mathrm{V} 1, \phi_{1, \mathrm{k}(\mathrm{x})}$ :

$$
\begin{aligned}
& \phi(x)=\sum_{k} l_{k} \phi_{i, k}(x) \\
& \psi(x)=\sum_{k} h_{k} \phi_{i, j}(x)
\end{aligned}
$$

The set $\left\{\phi_{j, k}(x)=2^{1 / 2} \phi\left(2^{j} x-k\right), k \in Z\right\}$ then forms a basis for $\mathrm{Wj}$, with $\mathrm{Wj}$ being the orthogonal complement to $\mathrm{Vj}$ and $\{\mathrm{Wj}\} \mathrm{j} \in \mathrm{Z}$ forming a basis for $\mathrm{L}^{2}(\mathrm{R})$. In practice, neither the scaling function nor the wavelet function is explicitly derived. Provided that the wavelet function has compact support, the scaling function is equivalent to a scaling filter and it is sufficient to determine the filter coefficients. The coefficients $l_{\mathrm{k}}$ in Eq. (5) 
form this scaling, or low-pass, filter and the coefficients $h_{k}$ in Eq. (6) form the wavelet, or high-pass, filter To ensure that a signal can be exactly reconstructed from its decomposition, the scaling coefficients and wavelet coefficients must form a quadrature mirror filter.
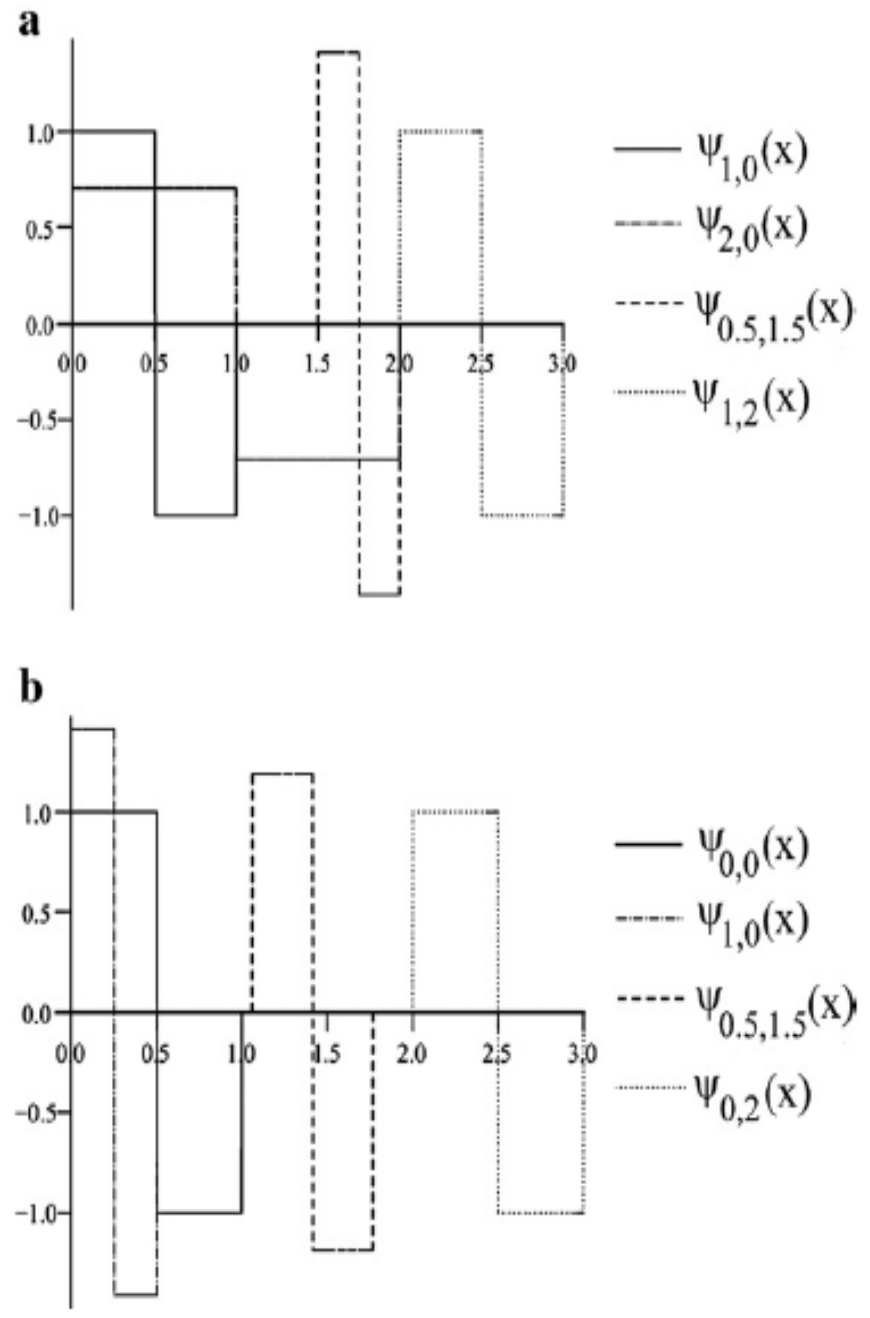

Figure 2. Mother wavelets and Daughter Wavelets
a. Daughter Wavelets according to equation 2
b. Daughter Wavelets according to equation 3

\section{DISCRETE WAVELET TRANSFORM}

Wavelet transforms provide a framework in which a signal is decomposed, with each level corresponding to a coarser resolution, or lower frequency band. There are two main groups of transforms, continuous and discrete. Discrete transforms are more commonly used and can be subdivided in various categories. Although a review of the literature produces a number of different names and approaches for wavelet transformations, most fall into one of the following three categories: decimated, un-decimated, and non-separated. A continuous wavelet transform is performed by applying an inner product to the signal and the wavelet functions. The dilation and translation factors are elements of the real line. For a particular dilation a and translation $b$, the wavelet coefficient $\mathrm{W}_{\mathrm{f}}(\mathrm{a}, \mathrm{b})$ for a signal $\mathrm{f}$ can be calculated as

$$
W_{f}(a, b)=\left\langle f, \psi_{a, b}\right\rangle=\int f(x) \psi_{a, b}(x) d x
$$

Wavelet coefficients represent the information contained in a signal at the corresponding dilation and translation. The original signal can be reconstructed by applying the inverse transform:

$$
f(x)=\frac{1}{c_{w}} \int_{-\infty-\infty}^{\infty} \int_{f}^{\infty} W_{f}(a, b) \psi_{a, b}(x) d b \frac{d a}{a^{2}}
$$

where $\mathrm{C} \psi$ is the normalization factor of the mother wavelet. Although the continuous wavelet transform is simple to describe mathematically, both the signal and the wavelet function must have closed forms, making it difficult or impractical to apply. The discrete wavelet is used instead. The term discrete wavelet transform (DWT) is a general term, encompassing several different methods. It must be noted that the signal itself is continuous; discrete refers to discrete sets of dilation and translation factors and discrete sampling of the signal. For simplicity, it will be assumed that the dilation and translation factors are chosen so as to have dyadic sampling, but the concepts can be extended to other choices of factors. At a given scale J, a finite number of translations are used in applying multi resolution analysis to obtain a finite number of scaling and wavelet coefficients. The signal can be represented in terms of these coefficients as

$$
f(x)=\sum_{k} C_{J k} \phi_{J k}(x)+\sum_{j=1}^{J} \sum_{k} d_{j k} \psi_{j k}(x)
$$

where $c_{\mathrm{Jk}}$ are the scaling coefficients and djk are the wavelet coefficients. The first term in Eq. (8) gives the low-resolution approximation of the signal while the second term gives the detailed information at resolutions from the original down to the current resolution J. The process of applying the DWT can be represented as a bank of filters, as in figure 3 . In case of a $2 \mathrm{D}$ image, a single level decomposition can be performed resulting in four different frequency bands namely LL, LH, HL and HH sub band and an $\mathrm{N}$ level decomposition can be performed resulting in $3 \mathrm{~N}+1$ different frequency bands and it is shown in figure 3 . At each level of decomposition, the image is split into high frequency and low frequency components; the low frequency components can be further decomposed until the desired resolution is reached. When multiple levels of decomposition are applied, the process is referred to as multi-resolution decomposition. In practice when wavelet decomposition is used for image fusion, one level of decomposition can be sufficient, but this depends on the ratio of the spatial resolutions of the images being fused. The conventional DWT can be applied using either a decimated or an un-decimated algorithm. In the decimated algorithm, the signal is down sampled after each level of transformation. In the case of a two-dimensional image, down-sampling is performed by keeping one out of every two rows and columns, making the transformed image one quarter of the original size and half the original resolution. The decimated algorithm can therefore be represented visually as a pyramid, where the spatial resolution becomes coarser as the image becomes smaller. The decimated algorithm is not shift-invariant, which means that it is sensitive to shifts of the input image. The decimation process also has a negative impact on the linear continuity of spatial features that do not have a 
horizontal or vertical orientation. These two factors tend to introduce artifacts when the algorithm is used in applications such as image fusion.

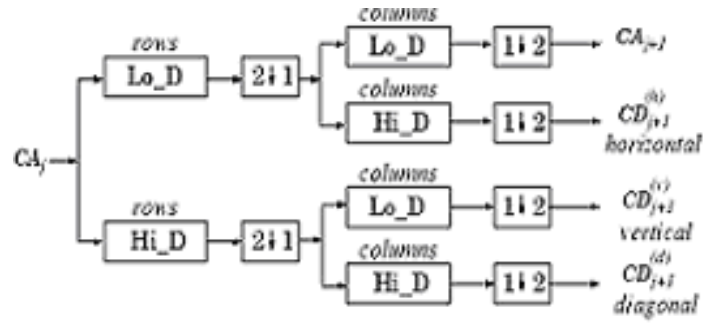

Where 2+1 Downsample columin: beep the enen injexed columss

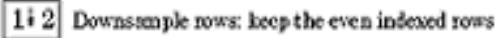
$\operatorname{mos}$ $\mathrm{X}$ Conrolve with filtet $\mathrm{X}$ the rows of the entry collewins

X Convolve wib filte: $\mathrm{X}$ the columas of the entry

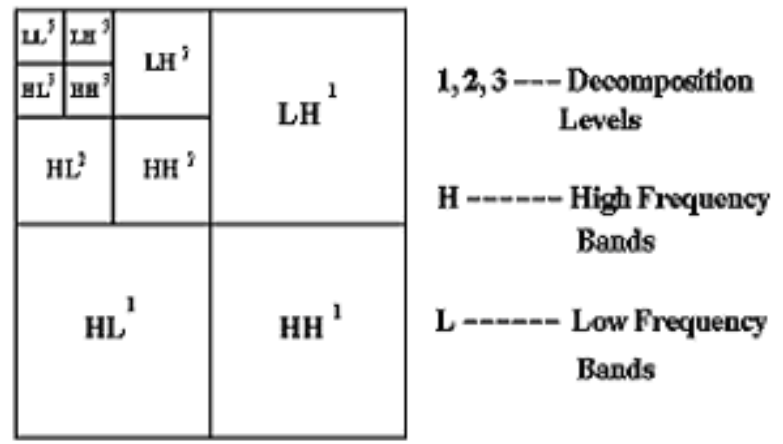

Figure 3. 2D-Discrete Wavelet Transform

\section{STATIONARY WAVELET TRANSFORM}

The Discrete Wavelet Transform is not a time- invariant transform. The way to restore the translation invariance is to average some slightly different DWT, called un-decimated DWT, to define the stationary wavelet transform (SWT). It does so by suppressing the down-sampling step of the decimated algorithm and instead up-sampling the filters by inserting zeros between the filter coefficients. Algorithms in which the filter is up-sampled are called "à trous", meaning "with holes". As with the decimated algorithm, the filters are applied first to the rows and then to the columns. In this case, however, although the four images produced (one approximation and three detail images) are at half the resolution of the original, they are the same size as the original image. The approximation images from the un-decimated algorithm are therefore represented as levels in a parallelepiped, with the spatial resolution becoming coarser at each higher level and the size remaining the same. The un-decimated algorithm is redundant, meaning some detail information may be retained in adjacent levels of transformation. It also requires more space to store the results of each level of transformation and, although it is shift-invariant, it does not resolve the problem of feature orientation. A previous level of approximation, resolution $\mathrm{J}-1$, can be reconstructed exactly by applying the inverse transform to all four images at resolution $\mathrm{J}$ and combining the resulting images. Essentially, the inverse transform involves the same steps as the forward transform, but they are applied in the reverse order. In the decimated case, this means up-sampling the approximation and detail images and applying reconstruction filters, which are inverses of the decomposition scaling and wavelet filters, first by columns and then by rows. For example, first the columns of the Vertical Detail image would be up-sampled and the inverse scaling filter would be applied, then the rows would be upsampled and the inverse wavelet filter would be applied. The original image is reconstructed by applying the inverse transform to each deconstructed level in turn, starting from the level at the coarsest resolution, until the original resolution is reached. Reconstruction in the un-decimated case is similar, except that instead of up-sampling the images, the filters are down-sampled before each application of the inverse filters. Shift-invariance is necessary in order to compare and combine wavelet coefficient images. Without shift-invariance, slight shifts in the input signal will produce variations in the wavelet coefficients that might introduce artifacts in the reconstructed image. Shift-variance is caused by the decimation process, and can be resolved by using the un-decimated algorithm. Let us recall that the DWT basic computational step is a convolution followed by decimation. The decimation retains even indexed elements. But the decimation could be carried out by choosing odd indexed elements instead of even indexed elements. This choice concerns every step of the decomposition process, so at every level we chose odd or even.

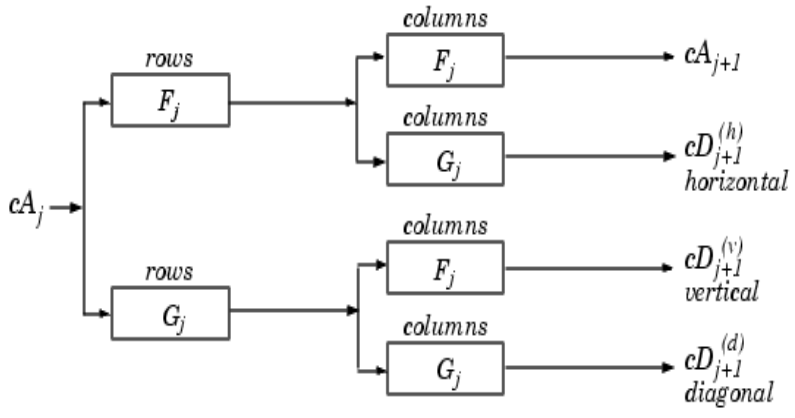

where

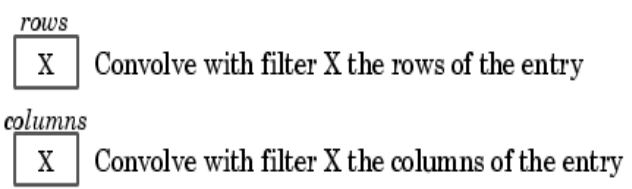

Figure 4. 2D Stationary Wavelet Transform.

If we perform all the different possible decompositions of the original signal, we have $2 \mathrm{~J}$ different decompositions, for a given maximum level $\mathrm{J}$. Let us denote by $\mathrm{j}=1$ or 0 the choice of odd or even indexed elements at step j. Every decomposition is labeled by a sequence of 0 's and 1 's: $=1, \mathrm{~J}$. This transform is called the decimated DWT. It is possible to calculate all the decimated DWT for a given signal of length $\mathrm{N}$, by computing the approximation and detail coefficients for every possible sequence. The SWT algorithm is very simple and is close to the DWT one. More precisely, for level 1, all the decimated DWT for a given signal can be obtained by convolving the signal with the appropriate filters as in the DWT case but without down sampling. Then the approximation and detail coefficients at level 1 are both of size $\mathrm{N}$, which is the signal length. The general step j convolves the approximation coefficients at level $\mathrm{j}-1$, with up sampled versions of the appropriate original filters, to produce the 
approximation and detail coefficients at level j. This can be visualized in the following figure 4 .

\section{MULTIWAVELET TRANSFORM}

Multiwavelets are very similar to wavelets but have some important differences. In particular, whereas wavelets have an associated scaling function $\varphi(t)$ and wavelet function $\psi(t)$, multiwavelets have two or more scaling and wavelet functions. For notational convenience, the set of scaling functions can be written using the vector notation $\varphi(t)=\left[\varphi_{1}(t) \varphi_{2}(t) \ldots \varphi_{r}(t)\right]^{T}$, where $\varphi(t)$ is called the multi scaling function. Likewise, the multiwavelet function is defined from the set of wavelet functions as $\psi(\mathrm{t})=\left[\begin{array}{llll}\psi_{1}(\mathrm{t}) & \psi_{2}(\mathrm{t}) & \ldots & \psi_{\mathrm{r}}(\mathrm{t})\end{array}\right]^{\mathrm{T}}$. When $\mathrm{r}=1, \psi(\mathrm{t})$ is called a scalar wavelet, or simply wavelet. While in principle $r$ can be arbitrarily large, the multiwavelets studied to date are primarily for $=2$.

$$
\begin{aligned}
& \phi(t)=\sqrt{2} \sum_{k=\infty}^{-\infty} H_{k} \phi(2 t-k) \\
& \psi(t)=\sqrt{2} \sum_{k=\infty}^{-\infty} G_{k} \phi(2 t-k)
\end{aligned}
$$

However, $\left\{\mathrm{H}_{\mathrm{k}}\right\}$ and $\left\{\mathrm{G}_{\mathrm{k}}\right\}$ are matrix filters, $\mathrm{H}_{\mathrm{k}}$ and $\mathrm{G}_{\mathrm{k}}$ are $\mathrm{r} \mathrm{x} \mathrm{r}$ matrices for each integer $\mathrm{k}$. The matrix elements in these filters provide more degrees of freedom than a traditional scalar wavelet. These extra degrees of freedom can be used to incorporate useful properties into the multiwavelet filters, such as orthogonality, symmetry, and high order of approximation. The key is to figure out how to make the best use of these extra degrees of freedom. During a single level of decomposition using discrete wavelet transform, the 2D image data is replaced with four blocks corresponding to the sub-bands representing either low pass or high pass filtering in each direction. The multi wavelet transform have two channels, so there will be two sets of scaling coefficients and two sets of wavelet coefficients.
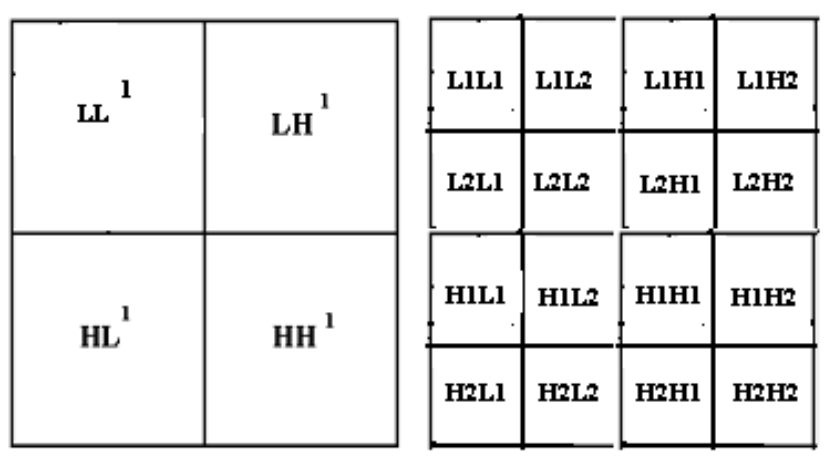

Figure 5. 2D Discrete and Multi Wavelet Transform

\section{LIFTING WAVELET TRANSFORM}

The conventional convolution-based implementation of the discrete wavelet transform has high computational and memory requirements. Recently, the lifting-based implementation of the discrete wavelet transform has been proposed to overcome these drawbacks and named as Lifting Wavelet Transform (LWT). LWT is also called second generation wavelet transform. It is a three-step filtering process: split, prediction and update. The original input signal is $f_{k}$. It is transformed into signal of high pass $h_{k}$ and low pass signal. $l_{k}$. In the split step, the original signal is split into two non-overlap subsets, namely even sequence and odd sequence. In the prediction step, even sequences are used to predict odd sequences. The prediction error forms the corresponding high-pass subband. In the update step, an approximation subband is obtained by updating even sequences with the scaled high-subband samples, which forms a low-pass subband. Backward transform is easy to find and has the same complexity as the forward transform. The two-step lifting transform can be generally described as

$$
\begin{aligned}
& h_{k}[x]=f_{2 k+1}[x]+\sum_{i} P_{i} f_{2(k-i)}[x] \\
& l_{k}[x]=f_{2 k}[x]+\sum_{j} u_{j} h_{k-j}[x]
\end{aligned}
$$

where $f_{k}[x]$ is the sequence of input data to be processed, $h_{k}$ and $l_{k}$ are resulting high-pass and low-pass sequences respectively, $p_{i}$ and $u_{j}$ are prediction and update coefficients of filters respectively.

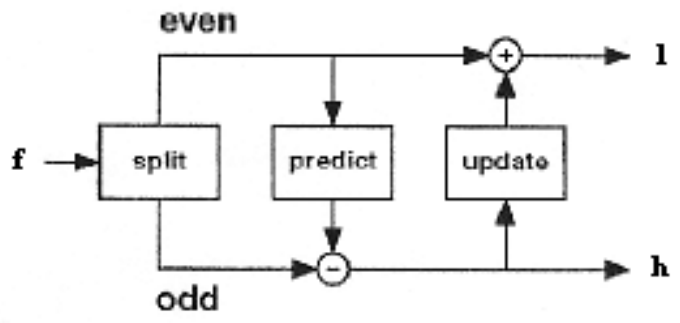

Figure 6. Lifting Wavelet Transform

\section{DUAL TREE WAVELET TRANSFORM}

The Dual Tree Wavelet Transform (DTWT) overcomes the limitations of DWT like poor directionality and shift invariance. It can be used to implement 2D wavelet transforms that are more selective with respect to orientation than is the separable 2D DWT. For example, the 2D DTWT transform produces six subbands at each scale, each of which is strongly oriented at distinct angles. There are two versions of the 2D DTWT transform namely Dual Tree Discrete Wavelet Transform (DTDWT) which is 2-times expansive, and Dual Tree Complex Wavelet Transform (DTCWT) which is 4-times expansive.

\section{Dual Tree Discrete Wavelet Transform}

The DTDWT of an image is implemented using two critically sampled separable DWT in parallel. Then for each pair of subbands, the sum and difference are taken. The six wavelets associated with DTDWT are illustrated in figure 2 as gray scale images. Note that each of the six wavelet are oriented in a distinct direction. Unlike the critically-sampled separable DWT, all of the wavelets are free of checker board artifact. Each subband of the 2D dual-tree transform corresponds to a specific orientation.

\section{Dual Tree Complex Wavelet Transform}

The DTCWT also gives rise to wavelets in six distinct directions and two wavelets in each direction. In each direction, one of the two wavelets can be interpreted as the real part of a complex valued wavelet, while the other wavelet can be interpreted as the imaginary part of a complex-valued wavelet. Because the complex version has twice as many wavelets as the real version of the transform, the complex version is 4-times expansive. The 
DTCWT transform is implemented as four critically sampled separable DWTs operating in parallel. However, different filter sets are used along the rows and columns. As in the real case, the sum and difference of subband images is performed to obtain the oriented wavelets. The twelve wavelets associated with the real 2D dual-tree DWT are illustrated in the following figure as gray scale images.

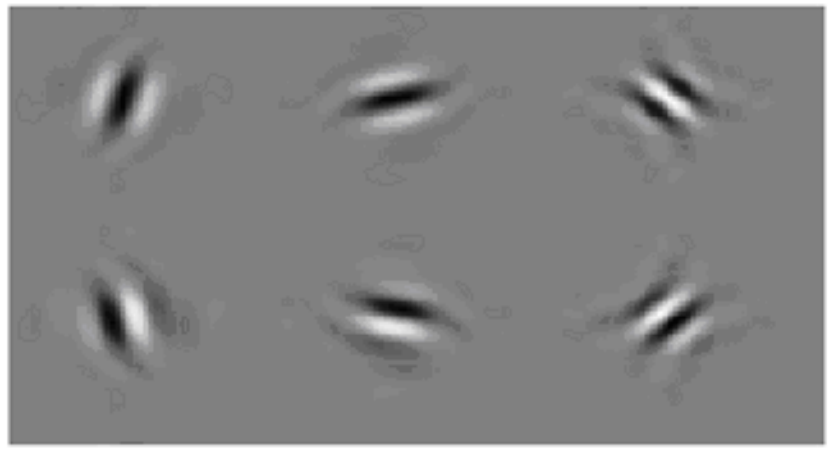

Figure 7. Directionality of DTDWT

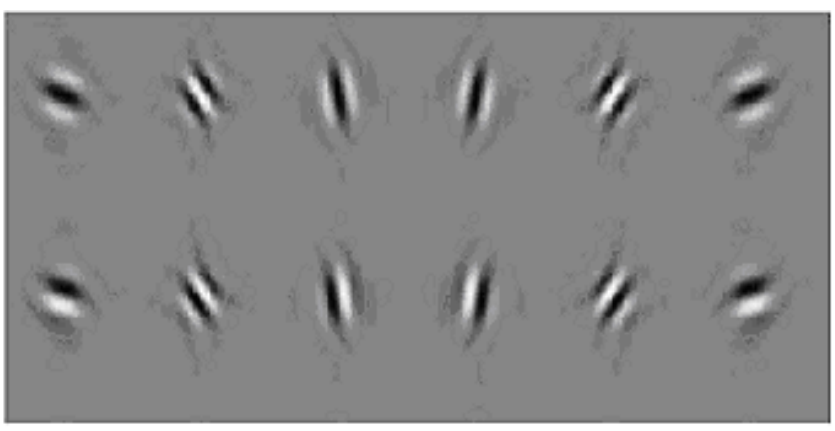

Figure 8. Directionality of DTCWT

The wavelets are oriented in the same six directions as those of DTDWT. However, there are two in each direction. If the six wavelets displayed on the first row are interpreted as the real part of complex wavelets, then the six wavelets displayed on the second row can be interpreted as the complex part of complex wavelets.

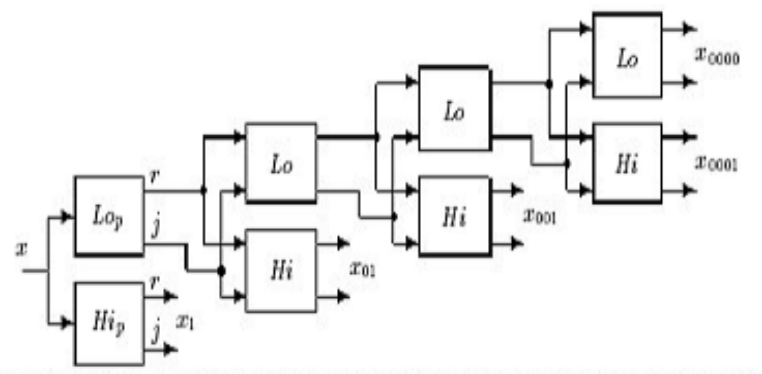

Figure 9. Four levels of Complex Wavelet Tree for real 1D input signal $x$

The filter bank structure of the DTCWT has CWT filters which have complex coefficients and generate complex output samples. This is shown in figure 9 , in which each block is a complex filter and includes down sampling by 2 (not shown) at its outputs. Since the output sampling rates are unchanged from the DWT, but each sample contains a real and imaginary part, a redundancy of $2: 1$ is introduced. The complex filters may be designed such that the magnitudes of their step responses vary slowly with input shift only the phases vary rapidly. The real part is an odd function while the imaginary part is even. The level 1 filters, Lop and Hip in figure 9 , include an additional pre filter, which has a zero at $\mathrm{z}$ $=-\mathrm{j}$, in order to simulate the effect of a filter tree extending further levels to the left of level 1. Extension of complex wavelets to 2-D is achieved by separable filtering along rows and then columns. However, if row and column filters both suppress negative frequencies, then only the first quadrant of the 2-D signal spectrum is retained. Two adjacent quadrants of the spectrum are required to represent fully a real 2-d signal, so we also need to filter with complex conjugates of either the row or column filters. This gives 4:1 redundancy in the transformed 2-D signal. If the signal exists in $m-d(m>2)$, then further conjugate pairs of filters are needed for each dimension leading to redundancy of $2^{\mathrm{m}}: 1$. The most computationally efficient way to achieve the pairs of conjugate filters is to maintain separate imaginary operators, $j_{1}$ and $\mathrm{j}_{2}$, for the row and column processing, as shown in figure 8 . This produces 4-element 'complex' vectors: $\left\{r, j_{1}, j_{2}, j_{1} j_{2}\right\}$ (where $r$ means 'real'). Each 4-vector can be converted into a pair of conventional complex 2-vectors, by letting $\mathrm{j}_{1}=\mathrm{j}_{2}=\mathrm{j}$ in one case and $j_{1}=-j_{2}=-j$ in the other case. This corresponds to sum and difference operations on the $\left\{r, \mathrm{j}_{1} \mathrm{j}_{2}\right\}$ and $\left\{\mathrm{j}_{1}, \mathrm{j}_{2}\right\}$ pairs in the summation blocks in figure 6 and produces two complex outputs, corresponding to first and second quadrant directional filters respectively. Complex filters in multiple dimensions provide true directional selectivity, despite being implemented separably, because they are still able to separate all parts of the m-D frequency space. For example a 2D DTCWT produces six band pass sub-images of complex coefficients at each level, which are strongly oriented at angles of $\pm 15^{\circ}, \pm 45^{\circ}, \pm 75^{\circ}$, shown by the double-headed arrows in figure 10.

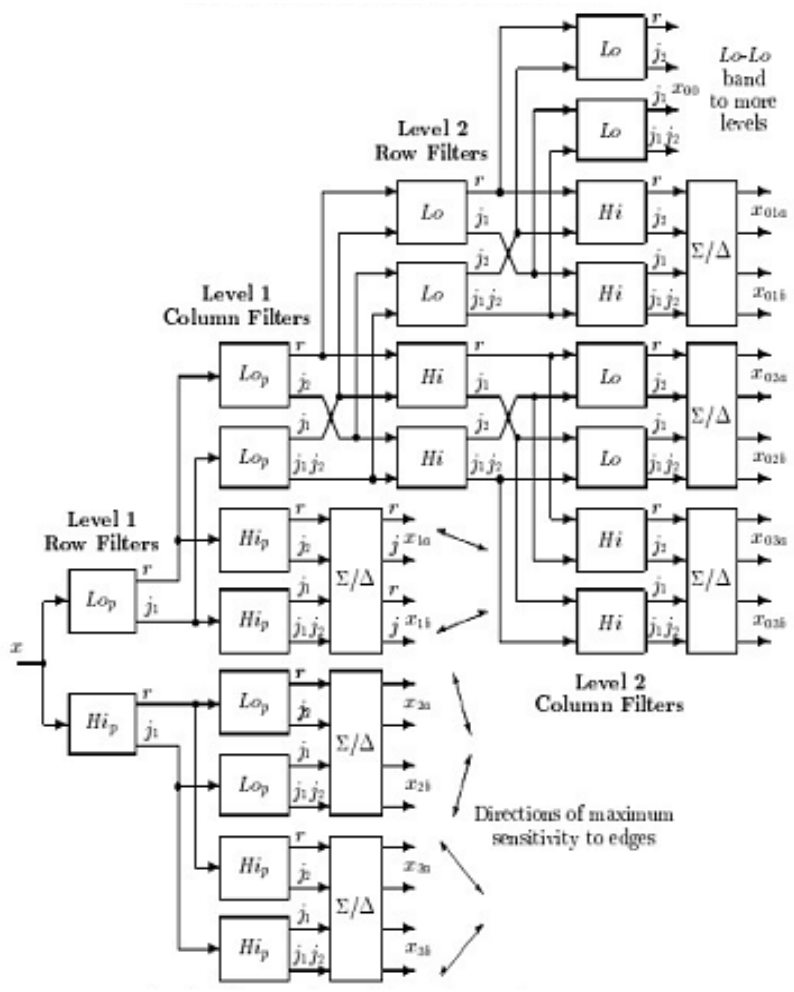

Figure 10. Two levels of the Complex Wavelet tree for a real 2-D input image $\mathrm{x}$ giving 6 directional bands at each level. 


\section{WAVELET BASED IMAGE FUSION}

Wavelet transform is first performed on each source images, and then a fusion decision map is generated based on a set of fusion rules. The fused wavelet coefficient map can be constructed from the wavelet coefficients of the source images according to the fusion decision map. Finally the fused image is obtained by performing the inverse wavelet transform. Let $\mathrm{A}(\mathrm{x}, \mathrm{y})$ and $\mathrm{B}(\mathrm{x}$, $\mathrm{y})$ are images to be fused, the decomposed low frequency sub images of $\mathrm{A}(\mathrm{x}, \mathrm{y})$ and $\mathrm{B}(\mathrm{x}, \mathrm{y})$ be respectively $l \mathrm{~A}_{\mathrm{J}}(\mathrm{x}, \mathrm{y})$ and $l \mathrm{~B}_{\mathrm{J}}$ $(\mathrm{x}, \mathrm{y})(\mathrm{J}$ is the parameter of resolution) and the decomposed high frequency sub images of $\mathrm{A}(\mathrm{x}, \mathrm{y})$ and $\mathrm{B}(\mathrm{x}, \mathrm{y})$ are $h \mathrm{~A}_{\mathrm{j}}^{\mathrm{k}}(\mathrm{x}, \mathrm{y})$ and $h \mathrm{~B}_{\mathrm{j}}^{\mathrm{k}}(\mathrm{x}, \mathrm{y}) .(\mathrm{j}$ is the parameter of resolution and $\mathrm{j}=1,2,3 \ldots J$ for every $j, \mathrm{k}=1,2,3 .$.$) . Then, the fused high and low frequency sub-$ images $F_{j}^{k}(x, y)$ are given as $F_{j}^{k}(x, y)=A_{j}^{k}(x, y)$ if $G\left(A_{j}^{k}(x, y)\right)$ $>=\mathrm{G}\left(\mathrm{B}_{\mathrm{j}}^{\mathrm{k}}(\mathrm{x}, \mathrm{y})\right)$, else $\mathrm{F}_{\mathrm{j}}^{\mathrm{k}}(\mathrm{x}, \mathrm{y})=\mathrm{B}_{\mathrm{j}}^{\mathrm{k}}(\mathrm{x}, \mathrm{y})$ and $\mathrm{F}_{\mathrm{J}}(\mathrm{x}, \mathrm{y})=l \mathrm{~A}_{\mathrm{J}}(\mathrm{x}$, $y)$ if $\mathrm{G}\left(\mathrm{A}_{\mathrm{J}}(\mathrm{x}, \mathrm{y})\right)>=\mathrm{G}\left(\mathrm{B}_{\mathrm{J}}(\mathrm{x}, \mathrm{y})\right)$, else $\mathrm{F}_{\mathrm{J}}(\mathrm{x}, \mathrm{y})=l B_{J}(\mathrm{x}, \mathrm{y})$ where $\mathrm{G}$ is the activity measure and $\mathrm{F}_{\mathrm{j}}^{\mathrm{k}}(\mathrm{x}, \mathrm{y}) \& \mathrm{~F}_{\mathrm{J}}(\mathrm{x}, \mathrm{y})$ are used to reconstruct the fused image $\mathrm{F}^{\prime}(\mathrm{x}, \mathrm{y})$ using the inverse wavelet transform. The block diagram representing the wavelet based image fusion is shown in figure 11.

\section{LEVELS IMAGE FUSION}

Activity measure for image fusion is calculated using three levels namely pixel level, area level and region level.

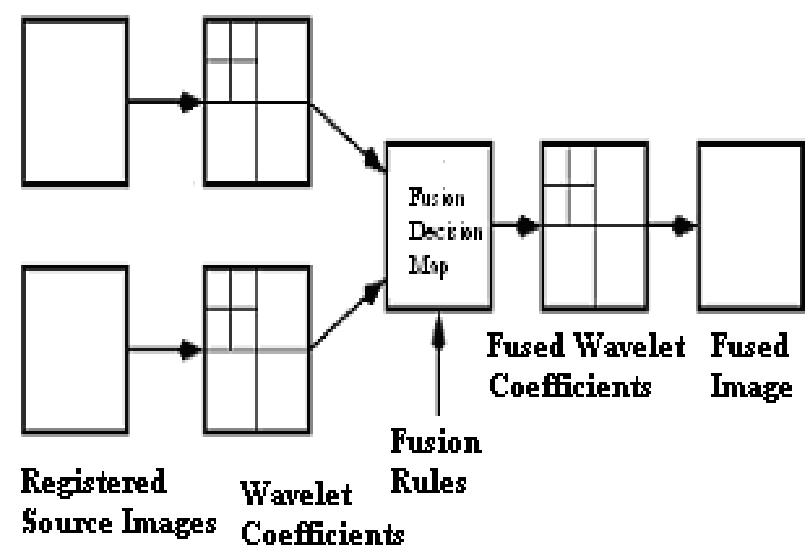

Figure 11. Wavelet Based Image Fusion.

Pixel level fusion uses the activity measure of pixel of interest to select the pixel to form the fused wavelet coefficients. In Pixel level fusion, we can take the average value of the pixel from the particular location of both the source images to form the pixel value of the fused image. However when this method is applied, there is chances of reduction of contrast of features uniquely presented in either of the images. As an alternative, the pixel which is having the highest gray level can be selected to form the fused image. Limitation is in the fusion of patterns that have roughly equal salience but opposite contrast, which results pathological [1]. Since larger absolute transform coefficients correspond to sharper brightness changes, the good integration rule is to select, at every point in the transform domain, the coefficients whose absolute values are higher [2].

Since the useful features in the image usually are larger than one pixel, the pixel by pixel selection rule of pixel level fusion may not be the most appropriate method. In area level of fusion algorithm, an area based selection rule is used. The images are first decomposed into sub bands using wavelet transform. Then the feature of each image patch over $3 \times 3$ or $5 \times 5$ window is computed as an activity measure associated with the pixel centered in the window[2]. This paper uses maximum absolute value over $3 \times 3$ window as the activity measure to select the pixel from source images to form the fused wavelet coefficient.

Region level image fusion, the activity measure is calculated over the region after the source images are segmented into various regions[5]. After creating the pyramid image using a wavelet transform, canny edge detector is applied to the lowest resolution approximation sub band of the image. After the edge detection, region segmentation is performed based on the edge information using region labeling algorithm. In the labeled image, zero corresponds to the edges and other different value represents different regions in the image. The activity level of region $\mathrm{k}$ in source image ' $\mathrm{n}$ ', $\mathrm{Al}_{\mathrm{n}}(\mathrm{k})$ is given by

$$
\mathrm{Al}_{\mathrm{n}}(\mathrm{k})=\frac{1}{\mathrm{~N}_{\mathrm{K}}} \sum_{1 \leq \mathrm{j} \leq \mathrm{N}_{\mathrm{k}}} \mathrm{Pj}
$$

where $N_{k}$ is the total number of pixels in region $k, P_{j}$ is the activity intensity of pixel $\mathrm{j}$ in region $\mathrm{k}$, which is the absolute value of pixel $\mathrm{j}$ in that region. Next step is to produce the decision map. The size of the decision map is the same as the size of the region image, which is the same size as the approximation band in the wavelet coefficient map. Each pixel in the decision map corresponds to a set of wavelet coefficients in each frequency band of all decomposition levels. Once the decision map is determined the mapping is determined for all the wavelet coefficients. Suppose, there are two registered images A and B to be fused then the decision map will be a binary image. For each pixel in this image, assume that value " 1 " means image A should be used instead of image B. Likewise the value " 0 " means image B should be used instead of image A. If a given pixel in the decision map is a "1" the all the wavelet coefficients corresponding to this pixel are taken from image A. If the pixel is " 0 " all the wavelet coefficients corresponding to this pixel are taken from image B. For a specific pixel of the decision map, $\mathrm{P}(\mathrm{i}, \mathrm{j})$, this pixel may be 1 ).In region $\mathrm{m}$ of image $\mathrm{A}$, and in region $\mathrm{n}$ of image B. 2). An edge point in one image, and in certain region in the other image 3 ).an edge point in both image. The value of each pixel in decision map is assigned according to the criterions like small regions preferred over large regions, edge points preferred over non edges points, high activity-level preferred over low activity level, decision on nonedge points first and consider their neighbors when making the decision on edge points.

\section{EVALUATION CRITERIA}

There are four evaluation measures are used in this paper, namely Root Mean Square Error (RMSE), Peak Signal to Noise Ratio (PSNR), Quality Index (QI)[3] and Normalized Weighted Performance Metric (NWPM)[4] which are given in the equations $13,314,15 \& 16$ respectively.

$$
\begin{array}{r}
\mathrm{RMSE}=\sqrt{\frac{\sum_{i=1}^{N} \sum_{j=1}^{N}[R(i, j)-F(i, j)]^{2}}{N^{2}}} \\
\mathrm{PSNR}=10 \log _{10}(255)^{2} /(\mathrm{RMSE})^{2}(\mathrm{db}) \\
\mathrm{QI}=\frac{4 \sigma_{a b} a b}{\left(a^{2}+b^{2}\right)\left(\sigma_{a}{ }^{2}+\sigma_{b}{ }^{2}\right)}
\end{array}
$$




$$
N W P M=\frac{\sum \forall_{i, j} Q_{i j}^{A F} W_{i j}^{A}+Q_{i j}^{A F} W_{i j}^{B}}{\sum \forall_{i, j} W_{i j}^{A}+W_{i j}^{B}}
$$

where $\mathrm{A}$ and $\mathrm{B}$ are the input images, $\mathrm{R}$ is the reference image, $\mathrm{F}$ is the fused image, $a$ is the average value of $A, b$ is the average value of $\mathrm{B}, \mathrm{Q}^{\mathrm{AF}}(\mathrm{i}, \mathrm{j})$ and $\mathrm{Q}^{\mathrm{BF}}(\mathrm{i}, \mathrm{j})$ are the edge preservation values.

\section{EXPERIMENTAL WORK}

A pair of source images namely Pepsi of size $512 \times 512$ is taken. The pair of source images to be fused is assumed to be registered spatially. The images are wavelet transformed using the same wavelet, and transformed to the same number of levels. In this paper, "HAAR" base is used in all the methods. For taking the wavelet transform of the two images, readily available MATLAB routines are taken. In each sub-band, individual pixels of the two images are compared based on the fusion rule that serves as a measure of activity at that particular scale and space. A fused wavelet transform is created by taking pixels from that wavelet transform that shows greater activity at the level. The inverse wavelet transform is the fused image with clear focus on the whole image.

\section{RESULTS}

For the above mentioned method, image fusion is performed using DWT, SWT, MWT, LWT, DTDWT and DTCWT, their performance is measured in terms of Root Mean Square Errors, Peak Signal to Noise Ratio, Quality Index \& Normalized Weighted Performance Metric and the results are shown in figure 4 and tabulated in table 1 .

\section{CONCLUSION}

This paper presents the comparison of all levels of fusion of multi focused images using DWT, SWT, LWT, MWT, DTDWT and DTCWT in terms of various performance measures. DTCWT provides very good results both quantitatively and qualitatively for pixel level and area level fusion. LWT provides computationally efficient and better qualitative and quantitative results in region level fusion among DWT, SWT, MWT and LWT. Hence using these fusion methods, one can enhance the image with high geometric resolution.

\section{REFERENCES}

1. P. J. Burt and R. J. Kolczynski, "Enhanced image capture through image fusion", proceedings of the $4^{\text {th }}$ International Conference on Computer Vision, pp. 173-182, 1993.

2. H. Li, B.S. Manjunath, and S.K. Mitra, "Multi-sensor image fusion using the wavelet transform", Proceedings of the conference on 'Graphical Models and Image Processing', pp. 235-245, 1995.

3. Zhou Wang and Alan C. Bovik, "A Universal Image Quality Index", IEEE Signal Processing Letters, Vol. 9, No.3, pp. 8184, March,2002.

4. C.S. Xydeas and V. Petrovic, 2000," Objective Image Fusion Performance Measure”, Electronics Letter, Vol.36, N0.4, pp. 308-309.

5. Z. Zhang and R.S. Blum," Region based image fusion scheme for concealed weapon detection", in Proceeding of the $30^{\text {th }}$ conference on CICC, March 1997.
Table 1. Performance Comparison of Wavelets

\begin{tabular}{|c|c|c|c|c|c|c|}
\hline & \multicolumn{6}{|c|}{ Pixel Level Image Fusion } \\
\hline & MWT & DWT & SWT & LWT & DTDWT & DTCWT \\
\hline RMSE & 5.7611 & 5.7111 & 5.2932 & 5.158 & 4.3555 & 4.3438 \\
\hline PSNR & 32.9206 & 32.9964 & 33.6565 & 33.8812 & 35.35 & 35.3735 \\
\hline Ql & 0.9917 & 0.9919 & 0.993 & 0.9935 & 0.9953 & 0.9953 \\
\hline \multirow[t]{3}{*}{ NWPM } & 0.6712 & 0.6664 & 0.7193 & 0.6568 & 0.7344 & 0.7383 \\
\hline & \multicolumn{5}{|c|}{ Area Level Image Fusion } & \\
\hline & DWT & SWT & MWT & LWT & DTDWT & DTCWT \\
\hline RMSE & 4.6594 & 4.3469 & 4.1694 & 3.9621 & 3.7029 & 3.677 \\
\hline PSNR & 34.7642 & 35.3673 & 35.7293 & 36.1724 & 36.7599 & 36.8209 \\
\hline Ql & 0.995 & 0.9959 & 0.996 & 0.9963 & 0.9968 & 0.9968 \\
\hline \multirow[t]{7}{*}{ NWPM } & 0.7293 & 0.751 & 0.7471 & 0.7097 & 0.754 & 0.7599 \\
\hline & & \multicolumn{4}{|c|}{ Region Level Image Fusion } & \\
\hline & & DWT & SWT & MWT & LWT & \\
\hline & RMSE & 3.2603 & 2.8771 & 2.8547 & 2.7899 & \\
\hline & PSNR & 37.8656 & 38.9518 & 39.0195 & 39.2191 & \\
\hline & Ql & 0.9974 & 0.998 & 0.998 & 0.9981 & \\
\hline & NWPM & 0.7692 & 0.7772 & 0.7773 & 0.7841 & \\
\hline
\end{tabular}

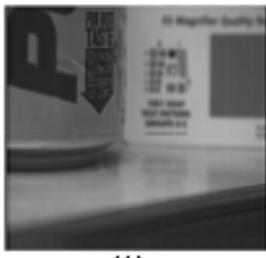

(A)

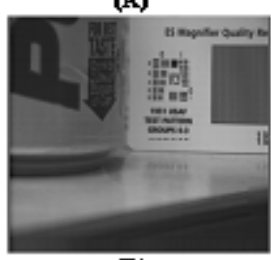

(D)

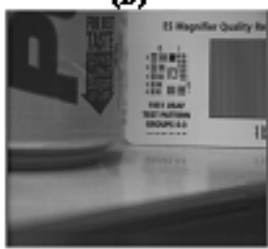

(G)

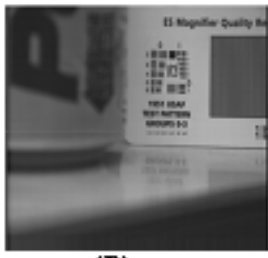

(B)

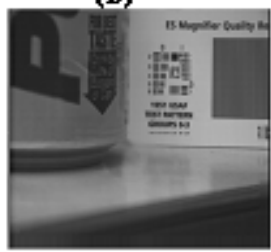

(E)

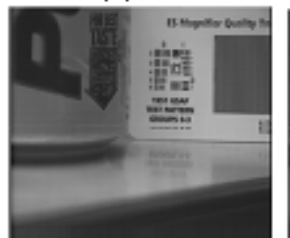

(H)

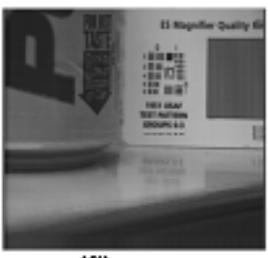

(C)

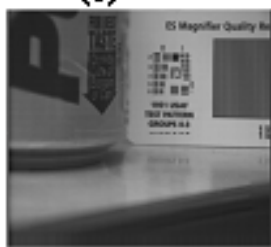

(F)

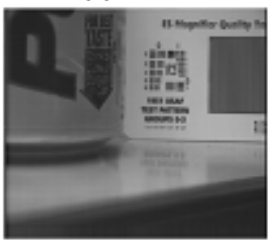

(I)
Figure 8. Results of Image Fusion Using Wavelets

a. Input Image 1 b. Input Image 2 c. Reference Image

d. Fused Image using DWT e. Fused Image using SWT

f. Fused Image using MWT g. Fused Image using LWT

h. Fused Image using DTDWT i. Fused Image using DTCWT 